American Journal of Biochemistry and Biotechnology 7 (4): 172-178, 2011

ISSN 1553-3468

(C) 2011 G. Wang et al., This open access article is distributed under a Creative Commons Attribution

(CC-BY) 3.0 license

\title{
High Incidence of Disorder-Promoting Amino Acids in the Amino Terminus of Mature Proteins in Bacillus subtilis
}

\author{
${ }^{1}$ Guangqiang Wang, ${ }^{1}$ Yining Dong, ${ }^{2}$ Haiqin Chen, \\ ${ }^{3}$ Heping Zhang, ${ }^{2}$ Yuanda Song, ${ }^{2}$ Hao Zhang and ${ }^{1}$ Wei Chen \\ ${ }^{1}$ State Key Laboratory of Food Science and Technology, \\ ${ }^{2}$ Centre for Food Biotechnology, School of Food Science and Technology, \\ Jiangnan University, Wuxi 214122, P.R. China \\ ${ }^{3}$ Department of Food Science and Engineering, College of Food Science and Engineering, \\ Inner Moneolia Agricultural University, Hohhot 010018, P.R. China
}

\begin{abstract}
Problem statement: Exported polypeptides are usually synthesized as preproteins which are composed with mature domains and the signal peptides. The foreign proteins are usually inefficiently secreted by the aid of signal peptides. Although we know that the mature protein played an important role in the protein export process, we do not know what properties of the mature domains are critical for protein export? This study explores the influence of the property of the mature domains on protein export in Bacillus subtilis. Approach: We analyzed the amino acid composition of 241 predicted exported proteins and 457 cytoplasmic proteins of Bacillus subtilis that have been previously reviewed using the program DAMBE, Codeprotein and Codeprotein Win. Results: Disorder-promoting amino acids in the first 14 residues of the mature domains were overrepresented in comparison to the residues in the $\mathrm{NH}_{2}$-terminal region of cytoplasmic proteins or the cytoplasmic proteins. Further, the $\mathrm{NH}_{2}$ terminus of the mature domain had more negatively charged residues and fewer positively charged residues than the $\mathrm{NH}_{2}$ terminus of the signal peptide. Conclusion/Recommendations: The presence of disordered domains in the $\mathrm{NH}_{2}$-terminal regions of mature domains may provide the binding site and maintain the exported proteins in a secretion-competent conformation and the contrary charged residues distribution in the two ends of hydrophobic core may help form the hairpin-like structure of the signal peptide. These findings may have important implications for the understanding of the contribution of mature domains to membrane targeting and to translocation.
\end{abstract}

Key words: Protein export, disorder-promoting amino acid, mature domain, signal peptide, Bacillus subtilis, hydrophobic core, charged residues, hairpin-like structure, disordered domains

\section{INTRODUCTION}

Exported proteins are initially synthesized as precursors with an amino-terminal signal peptide. These precursors are subsequently processed by a leader peptidase to yield the mature domains. The signal peptide can be divided into 3 distinct domains: a positively charged $\mathrm{N}$-terminus, which has been proposed to provide stable interactions with the negatively charged inner membrane phospholipids; a central hydrophobic core, which plays an important role in binding to the secretory components; and a cleavage site, which is processed by the respective signal peptidase (Fekkes and Driessen, 1999; Tjalsma et al., 2000).

Although the importance and roles of the signal peptides are generally accepted, only a signal peptide is not sufficient for the export of any fused protein. The foreign proteins are usually inefficiently secreted by the aid of signal peptides (Schein et al., 1986; Tommassen and Kroon, 1987), despite the fact that many of them are secretory proteins by nature (Simonen et al., 1992). Chen and Nagarajan (1993) have shown that the rate of secretion of barnase was improved by replacement of the barnase signal peptide with a heterologous signal peptide and the barnase signal peptide exported Escherichia coli alkaline phosphatase faster than mature barnase (Chen and Nagarajan, 1993). These facts suggested that some features of the mature protein played an important role in the protein export process. Indeed, some experimental work has shown that the membrane anchor sequences can block protein export (Davis and Model, 1985) and that the net charge of the

Corresponding Author: Wei Chen, State Key Laboratory of Food Science and Technology, Jiangnan University, Wuxi 214122, P.R. China 
$\mathrm{NH}_{2}$ terminus of the mature sequence affected protein translocation ( $\mathrm{Li}$ et al., 1988; Kajava et al., 2000). However, the latter rule was restricted to gram-negative bacteria, with no effect on gram-positive bacteria (Kajava et al., 2000). Furthermore, Gouridis have demonstrated that mature domains contained prominent targeting determinants and the targeting signals required for docking at the membrane were only presented on 'non-native' preproteins (Gouridis et al., 2009). Nevertheless, neither of those experiments answered the following question: what properties of the mature domains are critical for protein export? Currently, sufficient amino acid sequences are available to conduct detailed statistical studies on the property of the mature domains of exported proteins in Bacillus subtilis.

Although disordered regions lack rigid 3-D structures, they carry out various biological functions (Dunker et al., 2002; Patil and Nakamura, 2006; Salma et al., 2009). Dunker et al. (2002) identified twentyeight separate functions for 98 out of 115 disordered regions that included molecular recognition via binding to other proteins. Patil and Nakamura (2006) reported that disordered domains and high surface charge played a significant role in the binding ability of hubs. Hence disordered regions is important for protein-protein binding. It was not clear whether the binding site of mature proteins was related to the disordered domains during protein targeting. In this study, the amino acid composition of 241 predicted exported proteins and 457 cytoplasmic proteins of Bacillus subtilis was explored and revealed preference for disorder-promoting amino acids in the $\mathrm{NH}_{2}$-terminal regions of mature domains that may help understand the contribution of mature domains to membrane targeting.

\section{MATERIALS AND METHODS}

Generation of data sets: The sequence of every Open Reading Frame (ORF) of the genome of B. subtilis 168 was obtained via the National Center for Biotechnology Information (accession NC_000964). The exported proteins were taken from the previous survey of the secretome (Tjalsma et al., 2000). To reduce the number of false-positives, PhoD and YwbN, that were exported via the Tat pathway, anomalous exported proteins (those whose lengths of mature proteins were less 60 residues) and proteins with membrane-spanning domains annotated in the UniProtKB database were excluded. The proteins located in the cytoplasm and reviewed in the UniProtKB database were considered as cytoplasmic proteins. This resulted in a total of 241 exported proteins and 457 cytoplasmic proteins in B. subtilis. The folded proteins data set was compiled by Prilusky et al. (2005).

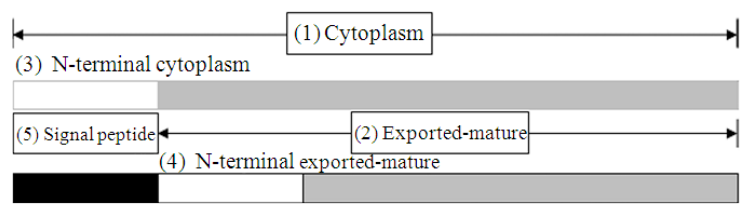

Fig. 1: Derivation of protein sequences comprising data sets 1-5. The top bar depicts cytoplamic proteins and the bottom bar depicts exported proteins. The regions of sequence depicted are not shown to scale

The amino acid sequences from the exported and cytoplasmic categories were obtained and used to derive 5 data sets (Fig. 1). Data set 1 (cytoplasm) was the amino acid sequences of cytoplasmic proteins. Data set 2 (exported-mature) was the mature domains of exported proteins. Data set 3 (N-terminal cytoplasm) was derived by copying an excerpt of the $\mathrm{N}$-terminal end of data set 1. Data set 4 (N-terminal exported-mature) was the $\mathrm{N}$ terminal end of data set 2 . Data set 5 (signal peptide) was the signal peptide of the exported proteins.

A more detailed analysis has provided information on the difference in the composition of ordered and disordered proteins and has enabled the classification of amino acids into 3 classes: order-promoting amino acids (Trp, Tyr, Phe, Ile, Leu, Val, Cys and Asn), disorder-promoting amino acids (Ala, Arg, Gly, Gln, Ser, Glu, Lys and Pro) and uncertain amino acids (His, Asp, Met and Thr) (Uversky and Dunker, 2010).

Analysis of data sets and statistical methods: For each data set, the mean percentage of amino acids was calculated using the program DAMBE (Xia and Xie, 2001), Codeprotein and CodeproteinWin (written in the Python programming language). For analysis, the first amino acid Met of all proteins (except proteins in the folded proteins data set) and the first amino acid Cys at the mature proteins of lipoproteins has been removed in the analysis process. The results were analyzed by oneway ANOVA. $\mathrm{R}$ version 2.7.1 was used for all statistical analysis.

\section{RESULTS}

Proportion of disordered residues in mature domains and cytoplasmic proteins: Table 1 shows the differences in the amino acid composition of the concatenated data sets. On average, the first 10 residues of data set 4 had $32.05 \%$ more disorder-promoting amino acids than the first 10 residues of data set 3 $(\mathrm{p}<2.2 \mathrm{e}-16)$, with $22.66 \%$ more compared to data set 1 $(\mathrm{p}<2.2 \mathrm{e}-16)$. But the proportion of disorder-promoting amino acids in data set 2 and data set 1 were virtually the same (48.90 and $48.81 \%$, respectively, $\mathrm{P}=0.1945$ ). 
Am. J. Biochem. \& Biotech., 7 (4): 172-178, 2011

Table 1: Amino acid composition in the data sets

\begin{tabular}{lllr}
\hline \multicolumn{1}{c}{ Data set } & $\begin{array}{l}\text { Order-promoting } \\
\text { acid (\%) }\end{array}$ & $\begin{array}{l}\text { Disorder-promoting } \\
\text { amino acids (\%) }\end{array}$ & $\begin{array}{r}\text { Uncertain amino } \\
\text { amino acids (\%) }\end{array}$ \\
\hline $\begin{array}{l}\text { (1) Cytoplasm } \\
\text { (2) Exported-mature }\end{array}$ & 35.10 & 48.81 & 16.06 \\
$\begin{array}{l}\text { (3) N-terminal cytoplasm } \\
\text { (the first ten) }\end{array}$ & 33.84 & 48.90 & 17.23 \\
$\begin{array}{l}\text { (3) N-terminal cytoplasm } \\
\text { (the first twenty) }\end{array}$ & 37.64 & 45.34 & 17.02 \\
$\begin{array}{l}\text { (4) N-terminal exported- } \\
\text { mature (the first ten) }\end{array}$ & 36.41 & 47.88 & 15.70 \\
$\begin{array}{l}\text { (4) N-terminal exported- } \\
\text { mature (the first twenty) } \\
\text { Genomic mean }\end{array}$ & 24.70 & 59.87 & 15.44 \\
\hline
\end{tabular}

${ }^{\mathrm{a}}$ : The percent of order-promoting amino acids were calculated using the the program DAMBE; ${ }^{\mathrm{b}}$ : The percent of order-promoting amino acids were calculated using the software Codeprotein

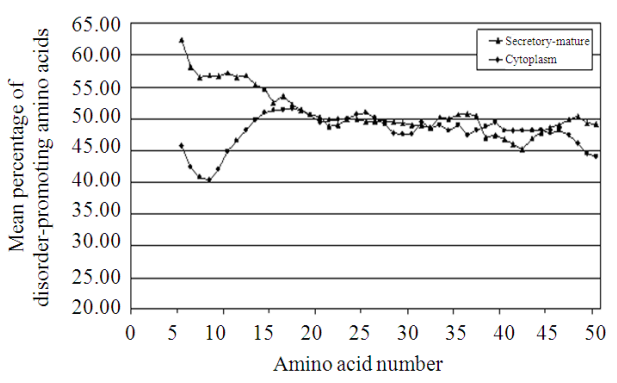

(a)

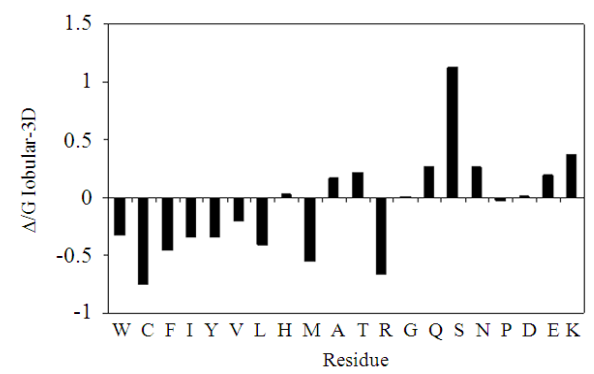

(b)

Fig. 2: (A) Mean percentage of disorder-promoting amino acids found in cytoplasmic proteins and exported mature domains. CodeproteinWin analysis of the first 50 amino acids of the sequences in the cytoplasmic proteins and exported mature domains, using a window size of 5. The amino acid number (terminal of window) is indicated on the $\mathrm{x}$-axis and the mean percentage of disordered residues are plotted on the y-axis. (B) Order/the first 14 residues of mature domains composition profile. Comparisons of amino acid compositions of ordered protein with the first 14 residues of mature domains. The ordinates are $(\%$ amino acid in the first 14 residues of mature domains$\%$ amino acid in ordered dataset) $/(\%$ amino acid in ordered dataset $)=\Delta$ /Globular $-3 \mathrm{D}$
The results indicated that the $\mathrm{NH}_{2}$-terminal regions of the mature domains had more disordered residues.

However, the trend seen in the comparison of data set 4 and data set 3 or data set 1 becomes weaker as the length of data set 4 and data set 3 becomes longer. For example, the first 20 residues of data set 4 have only $16.17 \%$ more disordered residues than data set 3 (Table $1, \mathrm{p}<2.2 \mathrm{e}-16)$. To explore whether the significance resulted from the difference in the early $\mathrm{NH}_{2}$ terminus of proteins and where these disordered residues are located within the sequences examined, the small software named CodeproteinWin was written in Python to analysis of the first 100 residues of the sequences of secretory protein and cytoplasmic proteins, using a window size of 5 . The overall distributions of disordered residues in the cytoplasmic and exported groups of sequences are shown in Fig. 2A. The proportion of disordered residues is relatively higher at the $\mathrm{NH}_{2}$ terminus of the mature domains and the level of disordered residues rapidly declines from left to right. At a position number 11 (from residue 7 to residue 11), the $\mathrm{NH}_{2}$ terminus of the mature domains had a massive bias for disordered residues compared to the cytoplasmic protein ( $\mathrm{p}=1.356 \mathrm{e}-07)$. At number 12 and number 13 , disordered residues were also preferred ( $p=1.065 \mathrm{e}-05, \mathrm{p}=0.003945)$, as the length becomes longer, the probability of difference between mature proteins and cytoplasmic proteins is equal to or more than the significance level (at number 14, $\mathrm{p}=0.03726$, at number $15, \mathrm{p}=0.5772$, at number $17, \mathrm{p}=0.7045$ ). After number 14, the mean percentage of disordered residues in the cytoplasmic proteins and secretory proteins is virtually the same over the remainder of the plotted sequence (48.88 and $48.47 \%$, respectively, $\mathrm{p}=$ $0.3483)$. Therefore, the first 14 residues of the mature domains were mainly composed of disordered residues.

The difference in the amino acid propensities of the first 14 residues of the mature domains and ordered proteins is shown in Fig. 2B. 


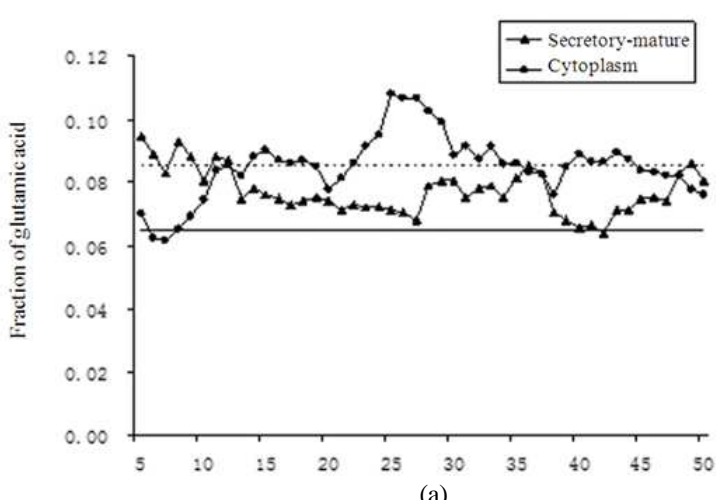

(a)

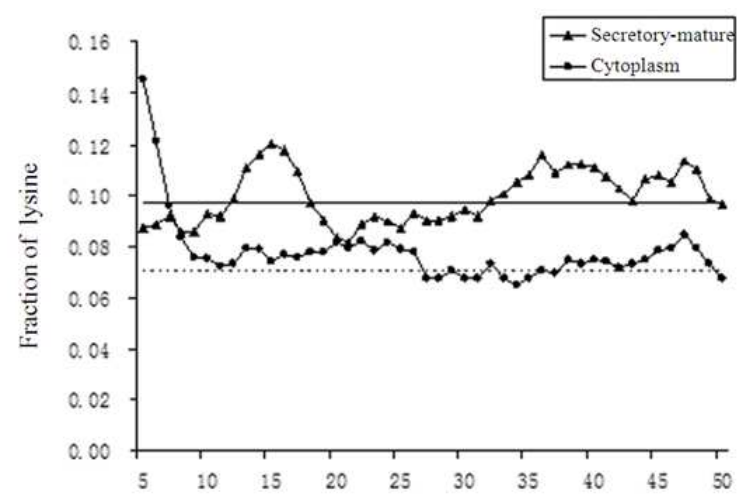

(c)

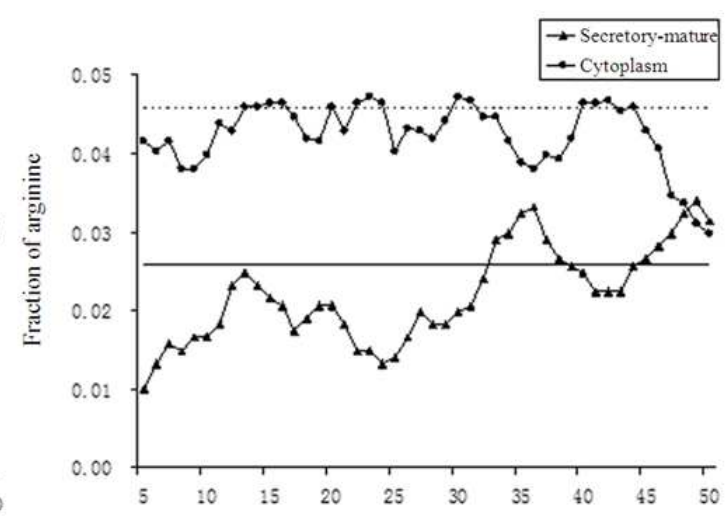

(b)

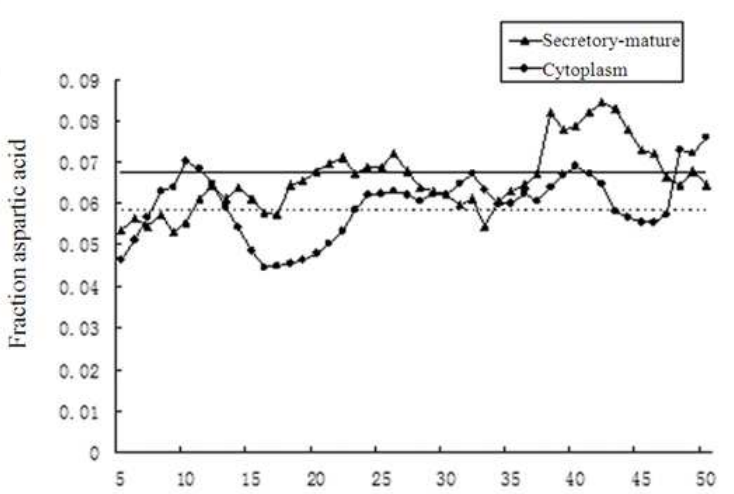

(d)

Fig. 3: CodeproteinWin analysis of the first 50 residues of the mature sequences and cytoplasmic proteins, using a window size of 5 . The $x$-axis reports the amino acid number of the terminal of the window and the mean percentage of the amino acid are plotted on the y-axis. The solid line and dashed line respectively represents the charged amino acid's percent composition in the mature domains and cytoplasmic proteins. A, mean percentage of glutamic acid in the first 50 residues of the mature sequences and cytoplasmic proteins; B, mean percentage of arginine; $\mathrm{C}$, mean percentage of lysine; $\mathrm{D}$, mean percentage of aspartic acid

Except for Met, Arg and Pro, the others showed the same trends in the order/disorder composition profile (Uversky and Dunker, 2010). The first 14 residues of the mature domains had more charged and polar residues like Glu, Ser, which were enriched in disordered proteins, but had less residues which were commonly found in ordered proteins, such as Trp, Cys, Phe (Dunker et al., 2001). These facts showed that the first 14 residues of the mature domains were difficult to fold into an ordered conformation and easily formed the disordered domains.

Analysis of distribution of charged residues in the $\mathbf{N H}_{2}$-terminal regions of mature domains: As shown in Fig. 3, the percentage of Glu in data set 4 was higher than that in data set 3 or data set 2 (for example, in the first 10 residues, 21.33 and $35.19 \%$ higher, $\mathrm{p}=$ 0.002044 and $\mathrm{p}=0.02495$, respectively) and the number of Arg in data set 4 was markedly lower than in data set 3 or data set 2 (for example, in the first 10 residues, 67.32 and $48.65 \%$ lower, $\mathrm{p}=5.266 \mathrm{e}-10$ and $\mathrm{p}$ $=4.747 \mathrm{e}-07$, respectively). Despite the abundance of Lys residues in data set 3 , especially in the first 5 residues, the number of Lys residues in data set 4 was not significantly different from that in data set 2 (for example, in the first 10 residues, $p=0.1441$ ). The number of Asp residues in data set 4 was not significantly different from that in data set $3(\mathrm{p}=$ 0.5134). These observations may implicate that the charged residues in the $\mathrm{NH}_{2}$-terminal regions of the mature domains are factors affecting protein export.

Moreover, as shown in Fig. 4, we compared the charged N-terminus of the signal peptide (with an average of 5 residues (Tjalsma et al., 2004) with the first 5 residues of the mature domains. 
Am. J. Biochem. \& Biotech., 7 (4): 172-178, 2011

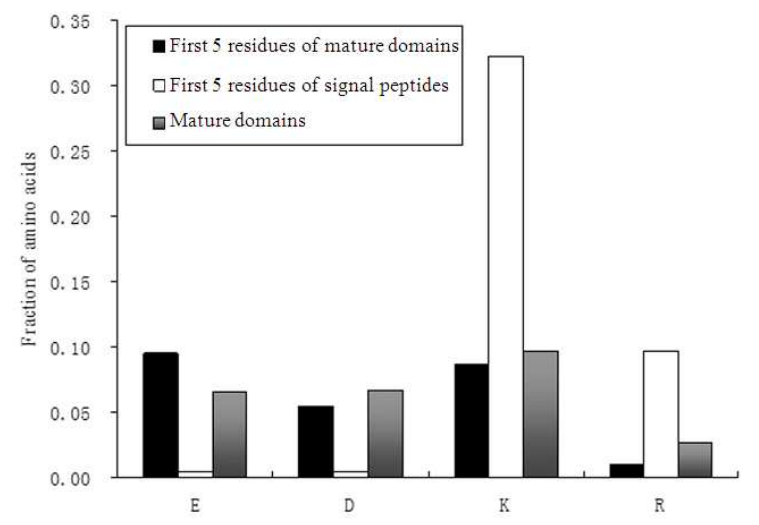

Fig. 4: Mean percentage of glutamic acid (E), aspartic acid (D), lysine (K) and arginine (R) found in the first 5 residues of mature domains (black), the first 5 residues of signal peptides (white) and mature domains (gray)

As confirmed by Heijne and Abrahmsen (1989), the $\mathrm{NH}_{2}$-terminus of the signal peptide contains more positively charged residues, especially Lys, but only few negatively charged residues.

However, the first 5 residues of the mature domains contained 18.1 times more negatively charged residues and 4.3 times less positively charged residues than the positively charged $\mathrm{N}$-domain. In addition, compared with the mature domains, the $\mathrm{NH}_{2}$-terminal regions of the mature domains had a tendency to contain more Glu and less Arg (Fig. 4), which mean the two ends of hydrophobic core had contrary charged residues distribution.

\section{DISCUSSION}

Although several experiments have suggested that the mature proteins are critical for protein export (Tommassen and Kroon, 1987; Simonen et al., 1992; Chen and Nagarajan, 1993; Davis and Model, 1985; Li et al., 1988; Kajava et al., 2000; Gouridis et al., 2009), the precise roles and the property of mature domains are unknown. In this study, we identified the amino acid composition of the mature domains in B. subtilis to study the property of the mature domains that facilitates their export.

The study has showed that the first 14 residues of the mature domains contained more disordered residues than the corresponding residues in the cytoplasmic proteins and mature domains. This was further corroborated in the comparison to the order/disorder composition profile (Uversky and Dunker, 2010). Surprisingly, Met, Arg and Pro were not as prevalent in these regions as in the disordered proteins. The depletion of Met may be caused by the analyzed mature proteins that did not contain the first Met (the folded proteins data set contained the first Met) and the roles of the N-terminus of mature domains may be related to the depletion of Arg and Pro.

It has been proved that the mature domain was the primary binding determinant in the targeting event (Gouridis et al., 2009). The presence of disordered domains in the $\mathrm{NH}_{2}$-terminal regions of mature domains can provide the binding site and some advantages for binding, such as fast speed of interaction (Shoemaker et al., 2000) or interaction with multiple proteins (Patil and Nakamura, 2006). Because Pro may hinder the diorder-to-order transition that many proteinprotein binding arose from (Patil and Nakamura, 2006; Uversky and Dunker, 2010), the binding function of mature domains may result in the depletion of Pro. The other role of the disordered domains in the $\mathrm{NH}_{2}$ terminal regions of the mature domains may be the maintenance of exported proteins in a secretioncompetent conformation for a long time to facilitate the interaction with the secretary components and protein export. Comparison to the $\mathrm{NH}_{2}$-terminal regions of the mature domains revealed that the $\mathrm{NH}_{2}$-terminal regions of the cytoplasm proteins have more order-promoting amino acids, so they can fold to the ordered globular structure quickly and then will not be recognized by protein export pathways. This feature may underly that besides the signal hypothesis (Manson, 1974), there is another mechanism that ensures that the cell efficiently recognizes the secretary proteins and cytoplasm proteins, which is the $\mathrm{NH}_{2}$-terminal regions of the mature domains enriching disorder-promoting amino acids while the $\mathrm{NH}_{2}$-terminal regions of the cytoplasm proteins containing a large number of order-promoting amino acids.

Apart from the disordered domains, we have shown that the $\mathrm{NH}_{2}$-terminal regions of the mature domains had more Glue and less Arg. Several different experiments have shown that the net charge of the amino terminus of the mature domains had either a neutral or negative net charge (Li et al., 1988; Kajava et al., 2000; Heijne, 1986), which only applied to gramnegative bacteria; neither eukaryotic nor gram-positive bacteria had this charge bias (Kajava et al., 2000). However, some experiments proved that a negatively charged N-terminus in the mature protein increased the secretion efficiency in Lactococcus lactis (Loir et al., 2001; Dieye et al., 2001). In our study, the $\mathrm{NH}_{2}-$ terminal regions of the mature domains had more Glu; thus, the presence of charged residues appeared to be more crucial than the net charge. 
Mutations in the positively charged $\mathrm{NH}_{2}$ region of the levansucrase signal peptide have shown that the secretion efficiency became slow when the 3 positive charges were reduced to 2 and the signal peptide was no longer processed when the charges were reduced to zero (Borchert and Nagarajan, 1991) and we showed the $\mathrm{NH}_{2}$-terminal regions of the mature domains had contrary charged residues to $\mathrm{NH}_{2}$-terminus of the signal peptide. These observations may implicate that the negatively charged residues in the $\mathrm{NH}_{2}$-terminal regions of the mature domains interacted with the positively charged N-terminus of the signal peptide. It was proposed that the signal peptide formed a hairpin-like structure that can insert into the membrane and unlooping of this hairpin can create a complete signal peptide that can insert into the membrane (Vrije et al., 1990). Our study suggested that the interaction between the $\mathrm{NH}_{2}$ terminal of the signal peptide and the $\mathrm{NH}_{2}$ terminal of the mature domains may help form the hairpin-like structure of the signal peptide. However, because the charged residues were not necessary for protein export in some secretory proteins (Chen and Nagarajan, 1994), it may be complementary to the role of the central hydrophobic core. The effect of the central hydrophobic core is the dominant factor in the formation of the hairpinlike structure.

By the help of the negatively charged residues in the $\mathrm{NH}_{2}$-terminal regions of the mature domains, the signal peptides form the hairpin-like structure that can insert into the membrane. The disordered domains of the mature domains may provide the primary binding site in targeting and docking events and can maintain the secretory proteins in a secretion-competent conformation for a long time that facilitates preproteins to interact with the secretory components. These features ensure efficient sorting of secretory proteins from cytoplasmic residents in the cells.

\section{CONCLUSION}

The amino acid composition of 241 predicted exported proteins and 457 cytoplasmic proteins of Bacillus subtilis was explored and we found that disorder-promoting amino acids in the first 14 residues of the mature domains were overrepresented in comparison to the amino acids in the cytoplasmic proteins or the NH2-terminal region. The finding may have important implications for the understanding of the contribution of mature domains to membrane targeting and to translocation.

\section{ACKNOWLEDGEMENT}

This study was supported by the National Nature Science Foundation of China (No 20836003, 30871952), the Science and Nature Foundation in Jiangsu Province (Grant No. BK2008103), the Fundamental Research Funds for the Central Universities JUSRP 11017 and the 111 Project B07029.

\section{REFERENCES}

Borchert, T.V. and V. Nagarajan, 1991. Effect of signal sequence alterations on export of levansucrase in Bacillus subtilis. J. Bacteriol., 173: 276-282.

Chen, M. and V. Nagarajan, 1993. The roles of signal peptide and mature protein in RNase (barnase) export from Bacillus subtilis. Mol. Gen. Genet., 239: 409-415. DOI: 10.1007/BF00276939

Chen, M. and V. Nagarajan, 1994. Effect of alteration of charged residues at the $\mathrm{N}$ termini of signal peptides on protein export in Bacillus subtilis. J. Bacteriol., 176: 5796-5801.

Davis, N.G. and P. Model, 1985. An artificial anchor domain: Hydrophobicity suffices to stop transfer. Cell, 41: 607-614. DOI: 10.1016/S00928674(85)80033-7

Dieye, Y., S. Usai, F. Clier, A. Gruss and J.C. Piard, 2001. Design of a protein-targeting system for lactic acid bacteria. J. Bacteriol., 183: 4157-4166. DOI: 10.1128/ JB.183.14.4157-4166.2001

Dunker, A.K., J.D. Lawson, C.J. Brown, R.M. Williams and P. Romero et al., 2001. Intrinsically disordered protein. J. Mol. Graph. Model., 19: 26-59. DOI: 10.1016/S1093-3263(00)00138-8

Dunker, A.K., C.J. Brown, J.D. Lawson, L.M. Iakoucheva and Z. Obradovic, 2002. Intrinsic disorder and protein function. Biochemistry, 41: 6573-6582. PMID: 12022860

Fekkes, P. and A.J. Driessen, 1999. Protein targeting to the bacterial cytoplasmic membrane. Microbiol. Mol. Biol. Rev., 63: 161-173. PMID: 10066835

Gouridis, G., S. Karamanou, I. Gelis, C.G. Kalodimos and A. Economou, 2009. Signal peptides are allosteric activators of the protein translocase. Nature, 462: 363-367. DOI: 10.1038/nature08559

Heijne, G.V. and L. Abrahmsen, 1989. Species-specific variation in signal peptide design Implications for protein secretion in foreign hosts. FEBS Lett., 244: 439-446. DOI: 10.1016/0014-5793(89)80579-4

Heijne, G.V., 1986. Net N-C charge imbalance may be important for signal sequence function in bacteria. J. Mol. Biol., 192: 287-290. DOI: 10.1016/00222836(86)90365-7 
Kajava, A.V., S.N. Zolov, A.E. Kalinin and M.A. Nesmeyanova, 2000. The net charge of the first 18 residues of the mature sequence affects protein translocation across the cytoplasmic membrane of gram-negative bacteria. J. Bacteriol., 182: 21632169. DOI: $10.1128 / J B .182 .8 .2163-2169.2000$

Li, P., J. Beckwith and H. Inouye, 1988. Alteration of the amino terminus of the mature sequence of a periplasmic protein can severely affect protein export in Escherichia coli. Proc. Nat. Acad. Sci. USA., 85: 7685-7689. PMID: 3051001

Loir, Y.L., S. Nouaille, J. Commissaire, L. Bretigny and A. Gruss et al., 2001. Signal peptide and propeptide optimization for heterologous protein secretion in Lactococcus lactis. Applied Environ. Microbiol., 67: 4119-4127. DOI: 10.1128/AEM.67.9.41194127.2001

Manson, L.A., 1974. Biomembranes. 1st Edn., Plenum Press, New York, ISBN: 0306398915.

Patil, A. and H. Nakamura, 2006. Disordered domains and high surface charge confer hubs with the ability to interact with multiple proteins in interaction networks. FEBS Lett. 580: 2041-2045. DOI: 10.1016/j.febslet.2006.03.003

Prilusky, J., C.E. Felder, T. Zeev-Ben-Mordehai, E.H. Rydberg and O. Man et al., 2005. FoldIndex(): A simple tool to predict whether a given protein sequence is intrinsically unfolded. Bioinformatics, 21: $\quad 3435-3438$.

DOI: 10.1093/bioinformatics/bti537

Salma, P., C. Chhatbar and S. Seshadri, 2009. Intrinsically unstructured proteins: Potential targets for drug discovery. Am. J. Infect. Dis., 5: 126-134. DOI: 10.3844/ajidsp.2009.126.134

Schein, C.H., K. Kashiwagi, A. Fujisawa and C. Weissmann, 1986. Secretion of mature IFN- $\alpha 2$ and accumulation of uncleaved precursor by Bacillus subtilis transformed with a hybrid $\alpha$-amylase signal sequence-IFN- $\alpha 2$ gene. Nat. Biotechnol., 4: 719725. DOI: $10.1038 /$ nbt0886-719
Shoemaker, B.A., J.J. Portman and P.G. Wolynes, 2000. Speeding molecular recognition by using the folding funnel: The fly-casting mechanism. Proc. Natl. Acad. Sci. USA., 97: 8868-8873. DOI: 10.1073/pnas.160259697

Simonen, M., E. Tarkka, R. Puohiniemi and M. Sarvas, 1992. Incompatibility of outer membrane proteins OmpA and OmpF of Escherichia coli with secretion in Bacillus subtilis: Fusions with secretable peptides. FEMS Microbiol. Lett., 79: 233-241. DOI: 10.1016/0378-1097(92)90215-A

Tjalsma, H., A. Bolhuis, J.D.H. Jongbloed, S. Bron and J.M.V. Dijl, 2000. Signal peptide-dependent protein transport in Bacillus subtilis: A genome-based survey of the secretome. Microbiol. Mol. Biol. Rev., 64: 515547. DOI: 10.1128/MMBR.64.3.515-547.2000

Tjalsma, H., H. Antelmann, J.D.H. Jongbloed, P.G. Braun and E. Darmon et al., 2004. Proteomics of protein secretion by Bacillus subtilis: Separating the "Secrets" of the secretome. Microbiol. Mol. Biol. Rev., 68: 207-233. DOI: 10.1128/ MMBR.68.2.207-233.2004

Tommassen, J. and T.D. Kroon, 1987. Subcellular localization of a PhoE-LacZ fusion protein in $E$. coli by protease accessibility experiments reveals an inner-membrane-spanning form of the protein. FEBS Lett., 221: 226-230. DOI: 10.1016/00145793(87)80930-4

Uversky, V.N. and A.K. Dunker, 2010. Understanding protein non-folding. Biochim. Biophys. Acta (BBA)- Proteins Proteomics, 1804: 1231-1264. DOI: 10.1016/j.bbapap.2010.01.017

Vrije, G.J.D., A.M. Batenburg, J.A. Killian and B.D. Kruijff, 1990. Lipid involvement in protein translocation in Escherichia coli. Mol. Microbiol., 4: $\quad 143-150$. DOI: $10.1111 / \mathrm{j} .1365-$ 2958.1990.tb02024.x

Xia, X. and Z. Xie, 2001. DAMBE: Software package for data analysis in molecular biology and evolution. J. Hered., 92: 371-373. DOI: 10.1093/jhered/92.4.371 